\title{
Instrumental electrodermal conditioning in the monkey (Cebus albifrons): Acquisition and long-term retention
}

\author{
H. D. KIMMEL, A. F. BRENNAN, D. C. McLEOD, \\ M. S. RAICH, and L. I. SCHONFELD \\ University of South Florida, Tampa, Florida 33620
}

\begin{abstract}
Eight Cebus albifrons monkeys received 25 sessions of discriminative operant conditioning of the skin conductance response (SCR), with colored lights as discriminative stimuli and with Sidman avoidance (SS-40 sec, RS-40 sec) scheduled during one light and response-contingent shock during the other. Discriminative stimulus segments were separated by 30 -sec periods of time-out from shocks and lights. Two extinction sessions were run 3 months after training. Almost from the beginning of conditioning, the monkeys made significantly more unelicited skin conductance responses in the avoidance periods than in punishment. The monkeys' heart rates also increased significantly, but there was no difference between avoidance and punishment. SCR frequency during extinction continued to differentiate significantly between avoidance and punishment, and there was a significant increase in this differentiation from the last conditioning session to the first extinction session, but the difference then reduced in the second session. The results indicated that monkey's SCRs are influenced by instrumental reinforcement contingencies somewhat in the same fashion as those of humans.
\end{abstract}

The delivery of instrumental reinforcement contingent upon electrodermal responses of humans has been shown to result in response modifications resembling those obtained with instrumental reinforcement of skeletal behavior (Kimmel, 1974). Reward and avoidance procedures have generally resulted in increased response frequencies or magnitudes, and punishment and omission procedures have resulted in reduced response frequencies or magnitudes. The reliability of the results of this research is unchallenged. The purpose of the present research was explicitly empirical, i.e., extension of the human work to subhuman primates and utilization of aversive experimental procedures that would be ethically unacceptable with humans.

The conditioning paradigm employed was based upon a procedure described by Greene and Sutor (1971). They used two different visual discriminative stimuli and the reinforcement was escape from or avoidance of aversive interruptions of music that was presented to human subjects. During the presence of one visual stimulus, the music played continuously when interresponse times were less than $30 \mathrm{sec}$. If the subject failed to make a skin conductance response (SCR), interruptions in the music began to occur. When the other stimulus was present, some subjects received response-contingent punishment, with interrupted music made contingent upon the occurrence of an SCR.

This research was funded by Contract No. 17-76-6053, U.S. Army Medical Research and Development Command.
Every one of Greene and Sutor's eight subjects made more responses in the presence of the escapeavoidance stimulus than during the other stimulus. This difference increased from the first five sessions to the last five sessions in seven of the eight subjects. Greene and Sutor showed that unelicited electrodermal responses in humans can be modified by negative reinforcement, via a Sidman avoidance schedule and that discriminative stimulus control over operant electrodermal responding can be achieved.

A preliminary investigation of operant electrodermal conditioning in the monkey, Cebus albifrons, using a procedure analogous to that employed by Greene and Sutor (1971), has recently been conducted in our laboratory (Kimmel \& Burns, 1977). In this study, two monkeys received red and green visual discriminative stimuli signaling Sidman avoidance or punishment. During avoidance, shock was administered on an SS-40-sec schedule, with SCRs postponing the shock for $40 \mathrm{sec}$. During punishment, the shock was contingent upon the SCR. The animals made an average of $60 \%$ more SCRs during avoidance than during punishment. Their heart rates were elevated by about $40 \mathrm{bpm}$ above baseline.

Based upon Greene and Sutor's (1971) human data and the results of our own preliminary investigation, the present study tested the hypothesis that SCR frequency would be greater during the presence of the avoidance discriminative stimulus than during the presence of the punishment stimulus and that this discriminative operant control of electrodermal responding would also be observable following con- 
ditioning, when the instrumental contingencies were no longer in force and the animals had received no shocks for three months. Heart rates were also recorded but no specific a priori hypothesis regarding these measures were made.

\section{METHOD}

\section{Subjects}

Eight adult male Cebus albifrons monkeys served as subjects in the experiment. The animals were habituated to handling and sitting in the primate chair twice per week for 3 months prior to data collection. During the last month of this period, skin conductance electrodes were attached and the baseline data employed in the development of appropriate computer programming for the detection of skin conductance responses and filtering of movement artifacts were obtained. Two of the monkeys had previously served in the pilot study 1 year earlier, during which they received 16 conditioning sessions with the same discriminative stimuli and reinforcment contingencies.

\section{Apparatus}

The animal was restrained in a specially designed Plexiglas primate chair with neck, chest, and waist yokes which prevented manual contact with the skin electrodes but which permitted the animal to remain comfortable for at least $1 \mathrm{~h}$. The monkey's tail protruded through a hole in the back of the chair for attachment of the shock electrodes. The chair also contained attachments for restraining the animal's feet. The monkey's tail and upper chest regions were shaved prior to each day's data collection.

A bipolar electrodermal recording configuration was employed in which the monkey's two plantar foot surfaces were series resistors in a constant-voltage circuit, with an output proportional to skin conductance (Venables \& Christie, 1974). The electrodes were of the zinc-zinc sulfate type $\left(1.0 \mathrm{~cm}^{2}\right)$ in Teflon cups filled with saline electrode paste. The total constant voltage across the monkey's skin was $.5 \mathrm{~V} \mathrm{dc}$. The output of the skin conductance circuit was amplified by instrumentation operational amplifiers and digitized by a voltage-to-frequency converter. The squarewave output of the voltage-to-frequency converter was processed by a Data General Nova 2 computer, which was programmed to maintain a continuous search for unelicited SCRs utilizing time bins of $.33 \mathrm{sec}$ and a response criterion of two successive time bins of .13 micromhos of increase in conductance per $.33 \mathrm{sec}$. Slow drifts in basal conductance were compensated for manually in the conductance input circuit and appropriate entries periodically teletyped into the computer. All responses that met or exceeded the criterion value were counted and their amplitude scored by the computer.

The computer program also detected downward conductance changes of .4 micromhos or more which occurred within a time bin of $.1 \mathrm{sec}$. On the assumption that these decreases resulted from movement-produced reductions in conductance at the skinelectrode junction, each of these decreases in conductance was followed by $2.0 \mathrm{sec}$ of time-out from contingencies, to prevent "rebound" conductance increases from counting as instrumental responses.

The cardiac signal was transduced by two-needle ECG electrodes placed subdermally in the right and left upper chest regions and taped in place after examination of the signal indicated adequate placement. It was amplified by a Grass Model 5 polygraph and fed into the coil of a relay whose contacts delivered a de pulse to the computer coincident with each heart beat. The computer was programmed to count heart beats during predetermined perıods and to record sequences of interbeat intervals immediately following the onset of the discriminative stimuli. The computer also administered all experimental events. Backup polygraphic records of skin conductance and heart rate were also obtained in case of computer or Teletype failure.

The primate chair containing the monkey was placed inside of a sound-attenuated chamber, $86.4 \mathrm{~cm}$ wide, $73.7 \mathrm{~cm}$ deep, and $116.8 \mathrm{~cm}$ high, so that the animal's face was oriented towards a panel which displayed the manipulanda and the visual stimuli. The panel was approximately $36 \mathrm{~cm}$ from the animal's face. A dim houselight and a speaker which delivered an $80-\mathrm{dB}$ continuous white masking noise were located behind the monkey. The electric shock was $3.0 \mathrm{~mA}$ in intensity and had a duration of $.2 \mathrm{sec}$. It was administered to the animal's tail via $7-\mathrm{mm}$ stainless steel electrodes. All of the equipment, including computer and Teletype, was located in a room adjacent to that which contained the soundattenuated monkey chambers.

\section{Procedure}

After the animal was placed in the chair and the electrodes attached, a 10-min period elapsed for electrode stabilization and to permit the polygraphic record to be checked for proper psychophysiological signal reception and amplification. A random sequence of four avoidance and four punishment visual discriminative stimuli (red or green, via the primate press panel) was then administered, with interpolated periods of $30 \mathrm{sec}$ of timeout (no visual stimulus or shocks) between the avoidance and punishment segments. The computer program permitted a maximum of two consecutive avoidance or punishment segments, except when three were needed to complete a daily sequence of four of each type. The duration of the visual stimuli varied randomly among $3.33,4.33$, and $5.33 \mathrm{~min}$, with a mean of $4.33 \mathrm{~min}$. Session length was about $40 \mathrm{~min}$, on the average, not including the initial periods of $10 \mathrm{~min}$ for electrode stabilization or the time needed to prepare and remove the animal.

Twenty-five sessions were run, two per week with 1 day between sessions, either Monday and Wednesday or Tuesday and Thursday. Data from the first conditioning session were not processed by the computer, due to a programming error which was corrected prior to the second session. However, polygraphic records from four of the eight animals were available for manual scoring of SCRs in the first session. During the avoidance segments, a 40-sec shock-shock Sidman schedule was administered, with criterion SCRs postponing shock for $40 \mathrm{sec}$. During the punishment segments, response-contingent shock was administered whenever a criterion response was detected. Responses occurring within $5 \mathrm{sec}$ of shocks did not result in shock postponement during avoidance or shock-administration during punishment, on the assumption that they were elicited by the shock. These responses were also not counted as instrumental SCRs. No shocks were administered during the first $40 \mathrm{sec}$ of each avoidance and punishment segment so that psychophysiological data would be available in the periods immediately following the transition from time-out to the discriminative stimuli, prior to the introduction of shocks and contingencies.

Three months after the last conditioning session, two extinction test sessions were administered during a 1 week period. During these sessions, all of the electrodes were attached and the program of visual discriminative stimuli and time-out was administered as previously, but no shocks were presented.

\section{RESULTS}

\section{Acquisition}

Frequency of instrumental SCRs. Data from all but the first acquisition session were available for analysis, but only Sessions $2-7$ and $20-25$ are presented below (i.e., the first and last 6 of the 24 sessions that were fully processed by the computer). Equipment failures during some of the intermediate 


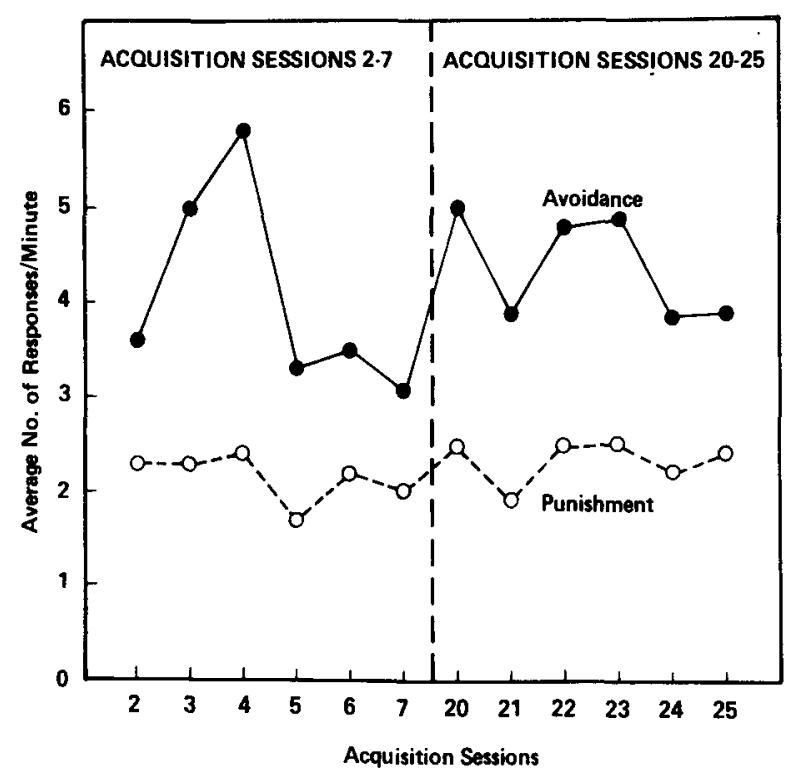

Figure 1. Average frequency of instrumental SCRs averaged for the four avoidance and four punishment segments in Acquisition Sessions 2-7 (early) and 20-25 (late).

sessions resulted in unavailability of data for some of the animals, but sufficient information is available in these two sets of 6 sessions to permit detailed examination of both early and late acquisition performance for all eight animals.

Figure 1 presents the average number of unelicited SCRs/min during the avoidance and punishment segments of Acquisition Sessions 2-7 and 20-25, excluding the initial contingency-free 40 -sec periods, which are considered separately below. As can be seen in Figure 1, the monkeys made approximately $60 \%$ more SCRs in the presence of the avoidance stimulus than in the presence of the punishment stimulus, and this difference was observable early in acquisition approximately to the same extent as it was near the end of acquisition. Analysis of variance of the SCR frequency data from these 12 sessions indicated that the overall avoidance-punishment difference was highly significant $[F(1,7)=14.56, p<$ $.01]$.

Because the avoidance-punishment difference appeared to be present even in the earliest acquisition sessions, a more detailed examination of instrumental responses during these sessions was undertaken. Figure 2 shows the average frequency of SCRs/min in each of the four alternating avoidance and punishment segments during Sessions 2, 3, and 4. As can be seen in Figure 2, the avoidance-punishment difference was smallest during Session 2 and increased over the next two sessions. A separate analysis of variance of the SCR data summarized in Figure 2 revealed that the avoidance-punishment difference was highly significant $[F(1,7)=14.51, p<.01]$ but the interaction with sessions was not $[\mathrm{F}(2,14)=2.67, \mathrm{p}<.20]$. Separate $t$ tests of the avoidance-punishment difference indicated that it was not separately significant during Session $2[\mathrm{t}(7)=1.16, \mathrm{p}<.30]$ but was significant during both Session 3 and Session 4 [t(7) $=2.44, \mathrm{p}<.04$, and $\mathrm{t}(7)=2.99, \mathrm{p}<.02$, respectively].

SCR frequency data during initial 40 -sec periods. The computer program automatically excluded the first $10 \mathrm{sec}$ after the onset of the discriminative stimuli and counted SCRs that occurred during the next $30 \mathrm{sec}$. This was done to exclude responses elicited by the onset of the discriminative stimuli. The average frequency of unelicited SCRs/min during these contingency-free periods increased from 4.2/min during Sessions 2-7 to 6.7/min during Sessions $20-25$, a change that was statistically significant $[F(1,7)=9.40, p<.025]$. There was no differentiation whatsoever between the avoidance and punishment periods, however, suggesting that the avoidance-punishment difference in SCRs/min that was seen after the contingencies began to be implemented (i.e., after the initial $40 \mathrm{sec}$ ) was probably not directly under the control of the discriminative stimuli. There was also a statistically significant tendency for these initial SCRs to increase in frequency across the successive stimulus segments during each daily experimental session $[\mathrm{F}(3,21)=3.53, \mathrm{p}<.05]$, but this effect was independent of the discriminative stimuli and did not change substantially from the early to the late conditioning sessions.

Heart rate. Heart rate was measured during the initial $40-\mathrm{sec}$ periods following the onset of each visual discriminative stimulus, before contingencies

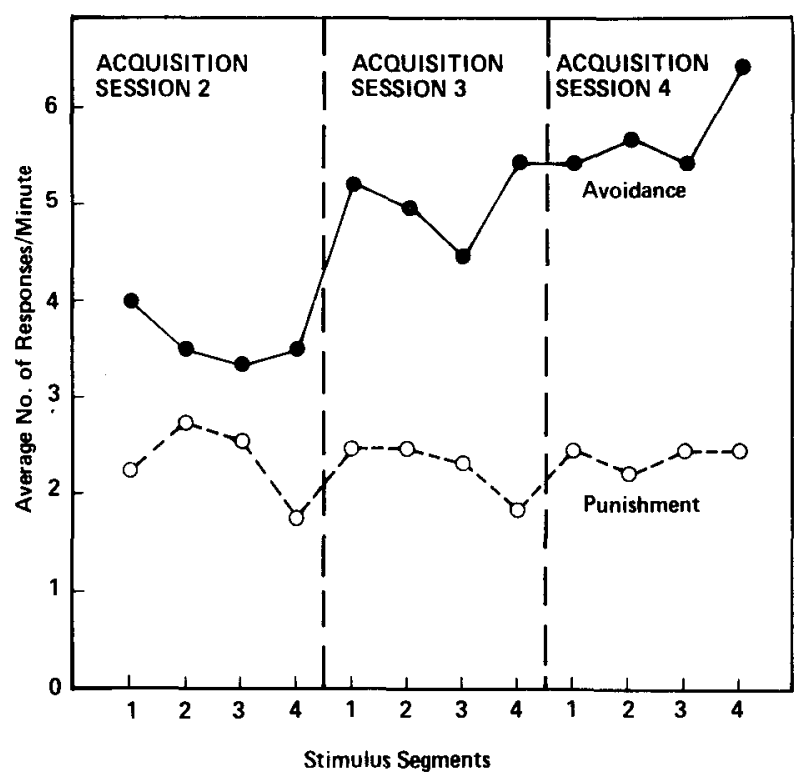

Figure 2. Average frequency of instrumental SCRs in each of the four avoidance and punishment segments of Acquisition Sessions 2, 3, and 4 . 
and shocks were introduced. The average interbeat interval over the last 50 beats of these 40 -sec periods was converted to beats/min for this purpose. There was a tendency for heart rate to increase within sessions, for both discriminative stimuli, during the early sessions. But this increase was not significant. During the last six sessions this within-session increase in heart rate occurred only during the punishment segments, but again the effect was not statistically significant.

Number of shocks administered. Overall, the animals received an average of .46 shocks/min during the avoidance segments and 2.24 shocks $/ \mathrm{min}$ during the punishment segments $[F(1,7)=15.97$, $\mathrm{p}<.01]$. The interaction between type of segment and early vs. late sessions was not statistically significant.

\section{Extinction}

Due to an electrical failure, the skin conductance signal from one animal was not recorded or processed by the computer during the first extinction session. This animal is excluded entirely from the extinction analysis.

Frequency of instrumental SCRs. Figure 3 presents the average number of unelicited SCRs/min during the presence of the avoidance and punishment discriminative stimuli for the last two acquisition sessions and the two extinction sessions. As is indicated in Figure 3, the difference in response frequency between avoidance and punishment that was present at the end of conditioning increased somewhat during extinction, especially during the first extinction session. Analysis of variance showed that the avoidance-punishment difference was significant when

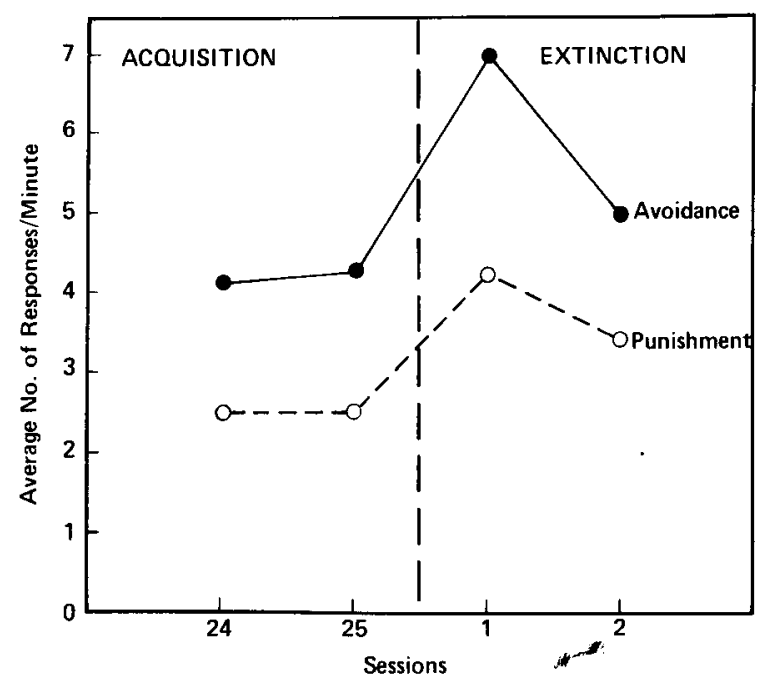

Figure 3. Average frequency of instrumental SCRs averaged for the four avoidance and four punishment segments in Acquisition Sessions 24 and 25 and Extinction Sessions 1 and $2(n=7)$. tested separately during extinction $[\mathrm{F}(1,6)=11.77$, $p<.025$ ] and when evaluated across the four sessions shown in Figure $3[F(1,6)=17.00, p<.01]$. A separate analysis of the transition from the 25 th conditioning session to the first extinction session showed that the interaction between the avoidancepunishment difference and the conditioning-extinction shift was also significant $[F(1,18)=10.68, p<.005]$. The increase in overall response frequency between the last two acquisition sessions and the two extinction sessions was not significant.

SCR frequency data during initial 40-sec periods. The number of unelicited SCRs/min at the beginning of the avoidance and punishment periods, when the contingencies had not yet begun, reflected the same overall increase as was seen in the instrumental response frequency data. As in acquisition, the difference between the avoidance and punishment segments was negligible in these measures. Analysis of variance revealed a significant interaction between extinction sessions and successive segments within session $[F(3,18)=3.87, p<.05]$, stemming from the fact that response frequency declined across segments during the first extinction session but not in the second. This was most noticeable in the avoidance segments of the first extinction session, but the implied interaction with type of segment was not significant.

\section{DISCUSSION}

The results of the present study are unambiguous in showing that reliable differentiation was established between the avoidance and punishment discriminative stimuli, in the sense that the animals made substantially more unelicited SCRs in the presence of the avoidance stimulus than during punishment. But because this difference appeared to be present almost from the very earliest conditioning sessions, detailed examination of the beginning sessions was undertaken to determine whether any evidence for gradual development of the difference could be discerned. As shown in Figure 2, the difference that was present during most of the conditioning sessions actually came into existence quite early and was maintained relatively constantly thereafter. It was not a statistically significant difference until the third session. The gradual manner in which the avoidance-punishment difference was observed to develop would seem to be consistent with the hypothesis that it was due to an instrumental conditioning process.

During the 40-sec periods at the beginning of each avoidance and punishment segment, when the discriminative stimulus had been turned on but no contingencies were operating and no shocks had been administered, the frequency of unelicited SCRs increased across conditioning sessions but did not 
differentiate between the two types of conditioning schedules. Since there was a significant difference in response frequency between avoidance and punishment when the contingencies were present and shocks were being administered, the absence of this difference during the initial, contingency-free periods may mean that the discriminative stimuli did not acquire direct control of responding but that the animals simply modified their response rates in relation to whether or not shocks were being administered. That is, during the avoidance segments the animal received a shock following $40 \mathrm{sec}$ of nonresponse. This shock may have served as a signal for responding thereafter, so that reduction of shock frequency during the remainder of that particular avoidance segment would result. But, at the beginning of a punishment segment, nonresponse did not result in shock, so that there may have been less likelihood that responding would increase in frequency during these periods. This is not what "stimulus control" usually means.

The heart rate data also failed to provide statistically significant evidence for differentiation between the stimuli signaling avoidance and punishment contingencies. The monkeys' differential pattern of responding resulted in a significantly greater number of shocks/minute occurring during the punishment segments as compared with avoidance, but this difference did not change significantly during conditioning. These results conform to previous findings of independence of autonomic effectors when instrumental contingencies are applied to one and more than one are observed (Kimmel \& Kimmel, 1967; Miller \& Banuazizi, 1968; Shapiro, Crider, \& Tursky, 1964).

The extinction results, of course, are also germane to the question of stimulus control of instrumental responding, since neither contingencies nor shocks were present in extinction. As shown in Figure 3, the avoidance-punishment difference in SCR frequency during the last two conditioning sessions persisted without reduction during the 3 months that intervened between the end of acquisition and the beginning of extinction. Indeed, there was a significant increase in the avoidance-punishment effect between the last conditioning session and the first extinction session 3 months later. Figure 3 also suggests that the differentiation declined slightly from the first to the second extinction session, but this reduction was not significant. In any case, the fact that the avoidancepunishment difference was still present during extinction indicates that the discriminative stimuli played a significant role in controlling the differentiation. It is of interest also that no differentiation between avoidance and punishment response frequencies was observed during the $40-\mathrm{sec}$ periods at the beginning of each segment during extinction, as was also true during conditioning. This suggests that the difference between the avoidance and punishment periods may not be manifested fully until the discriminative stimuli have been present for time periods longer than $40 \mathrm{sec}$.

In addition to providing support for the conclusion that discriminative stimulus control of SCR frequency was established by the conditioning procedure, the extinction results also can be taken to mean that instrumental electrodermal conditioning in the monkey produces quite persistent (i.e., "relatively permanent") behavioral changes. Thus, instrumental modification of autonomically mediated behavior is similar in this respect to instrumental modification of skeletal behavior, as should be true if the term "instrumental" means the same thing in both cases. The possiblity of skeletal mediation of the SCR results is not ruled out by the present data, although the manner in which the computer program detected abrupt, large decreases in skin conductance and initiated 2-sec periods of time-out following them probably reduced this influence somewhat. It made no difference whether an increase in skin conductance was due to simple rebound or reflected a "real" SCR that was reflexively elicited by the movement; in either case, the increase was not scored as a response and was not reinforced. Since only movements that actually influenced the contact between the skin conductance electrodes and the monkey's foot could initiate these times out and since even these movements had to be sufficiently abrupt and intense to result in a drop in conductance of .4 micromhos within .1 sec, the issue of skeletal mediation remains open.

\section{REFERENCES}

Greene, W. A., \& Sutor, L. T. Stimulus control of skin resistance responses on an escape-avoidance schedule. Journal of the Experimental Analysis of Behavior, 1971, 16, 269-274.

KIMMEL, H. D. Instrumental conditioning of autonomically mediated responses in human beings. American Psychologist, 1974,29, 325-335.

Kimmes, H. D., \& Burns, R. A. Inter-effector influences in operant autonomic control. In J. Beatty \& H. Legewie (Eds.), Biofeedback and behavior. New York: Plenum, 1977.

KIMMEL, H. D., \& KıмmEL, E. Inter-effector influences in operant autonomic conditioning. Psychonomic Science, 1967, 9, 191-192.

Miller, N. E., \& Banuazizi, A. Instrumental learning by curarized rats of a specific visceral response, intestinal or cardiac. Journal of Comparative and Physiological Psychology, $1968,65,1-7$.

Shapiro, D., Tursky, B., \& Crider, A. Differentiation of an autonomic response through operant reinforcement. Psychonomic Science, 1964, 1, 147-148.

Venabiff, P. H., \& Christie, M. J. Mechanisms, instrumentation, recording techniques, and quantification of response. In W. F. Prokasy \& D. G. Raskin (Eds.), Electrodermal activity in psychological research. New York: Academic Press, 1973.

(Received for publication December 7, 1978; revision accepted March 5, 1979.) 